\title{
HOEFFDING SPACES AND SPECHT MODULES
}

\author{
Giovanni Peccati ${ }^{1}$ And Jean-Renaud PyCKe ${ }^{2}$
}

\begin{abstract}
It is proved that each Hoeffding space associated with a random permutation (or, equivalently, with extractions without replacement from a finite population) carries an irreducible representation of the symmetric group, equivalent to a two-block Specht module.
\end{abstract}

Mathematics Subject Classification. 05E10, 60C05.

Received December 2, 2009. Revised June 18, 2010.

\section{INTRODUCTION}

Let $X_{(m)}=\left(X_{1}, \ldots, X_{m}\right)(m \geq 2)$ be a sample of random observations. According e.g. to [9], we say that $X_{(m)}$ is Hoeffding-decomposable if every symmetric statistic of $X_{(m)}$ can be written as an orthogonal sum of symmetric $U$-statistics with degenerated kernels of increasing orders. In the case where $X_{(m)}$ is composed of i.i.d. random variables, Hoeffding decompositions are a classic and very powerful tool for obtaining limit theorems, as $m \rightarrow \infty$, for sequences of general symmetric statistics of the vectors $X_{(m)}$. See e.g. [12], or the references indicated in the introduction to [9], for further discussions in this direction.

In recent years, several efforts have been made in order to provide a characterization of Hoeffding decompositions associated with exchangeable (and not necessarily independent) vectors of observations. See El-Dakkak and Peccati [7] and Peccati [9] for some general statements; see Bloznelis [2], Bloznelis and Götze [3,4] and Zhao and Chen [14] for a comprehensive analysis of Hoeffding decompositions associated with extractions without replacement from a finite population.

In the present note, we are interested in building a new explicit connection between the results of $[3,4,14]$ and the irreducible representations of the symmetric groups $\mathfrak{S}_{n}, n \geq 2$. In particular, our main result is the following.

Theorem 1.1. Let $1 \leq m \leq n / 2$, and let $X_{(m)}=(X(1), \ldots, X(m))$ be a random vector obtained as the first $m$ extractions without replacement from a population of $n$ individuals. For $l=1, \ldots, m$, let $S H_{l}$ be the lth symmetric Hoeffding space associated with $X_{(m)}$ (that is, $S_{H_{l}}$ is the vector space of all symmetric U-statistics with a completely degenerated kernel of order $l$ ). Then, for every $l=1, \ldots, m$, there exists an action of $\mathfrak{S}_{n}$ on $S H_{l}$, such that $S_{l}$ is an irreducible representation of $\mathfrak{S}_{n}$. This representation is equivalent to a Specht module of shape $(n-l, l)$.

\footnotetext{
Keywords and phrases. Exchangeability, finite population statistics, Hoeffding decompositions, irreducible representations, random permutations, Specht modules, symmetric group.

${ }^{1}$ Équipe Modal'X, Université Paris Ouest-Nanterre La Défense, 200 avenue de la République, 92000 Nanterre, France. giovanni.peccati@gmail.com

2 Département de Mathématiques, Université d'Évry, France. jrpycke@maths.univ-evry.fr
} 
We refer the reader to the forthcoming Section 2 for some basic results on the representations of the symmetric group and two-block Specht modules. We will see that Theorem 1.1 provides de facto a new probabilistic characterization of two-block Specht modules, as well as some original insights into the combinatorial structure of Hoeffding spaces. Observe that the case where $n / 2<m \leq n$ can be reduced to the framework of present paper by standard arguments (see for instance [3], Prop. 1). One should note that a connection between decompositions of symmetric statistics and representations of $\mathfrak{S}_{n}$ is already sketched in Diaconis' celebrated monograph [5]: in particular, the results of the present paper can be regarded as a probabilistic counterpart to the spectral analysis on homogeneous spaces developed in chapters 7 and 8 of [5].

The rest of this note is organized as follows. In Section 2 we provide some background on the representations of the symmetric group. Sections 3 and 4 focus, respectively, on uniform random permutations and Hoeffding spaces. Section 5 contains the statements and proofs of our main results.

\section{BACKGROUND}

For future reference, we recall that a $k$-block partition of the integer $n \geq 2$ is a $k$-dimensional vector of the type $\lambda=\left(\lambda_{1}, \ldots, \lambda_{k}\right)$, such that: (i) each $\lambda_{i}$ is a strictly positive integer, (ii) $\lambda_{i} \geq \lambda_{i+1}$, and (iii) $\lambda_{1}+\cdots+\lambda_{k}=n$. One sometimes writes $\lambda \vdash n$ to indicate that $\lambda$ is a partition of $n$.

We also write $[n]=\{1, \ldots, n\}$ to indicate the set of the first $n$ positive integers. Finally, given a finite set $A$, we denote by $\mathfrak{S}_{A}$ the group of all permutations of $A$, and we use the shorthand notation $\mathfrak{S}_{[n]}=\mathfrak{S}_{n}, n \geq 1$. In other words, when writing $x \in \mathfrak{S}_{A}$, we mean that

$$
x: A \rightarrow A: a \mapsto x(a)
$$

is a bijection from $A$ to itself.

\subsection{Some structures associated with two-block partitions}

We now introduce some classic definitions and notation related to tableaux and tabloids; see Sagan [11], Chapter 2 (from which we borrow most of our terminology and notational conventions) for any unexplained concept or result. For the rest of the section, we fix two integers $n$ and $m$, such that $1 \leq m \leq n / 2$. Observe that $n-m \geq m$, and therefore the vector $(n-m, m)$ is a two-block partition of the integer $n$.

Remark 2.1. It is sometimes useful to adopt a graphical representation of tableaux and tabloids by means of Ferrer diagrams. Since we uniquely deal with two-block tableaux and tabloids, and for the sake of brevity, in what follows we shall not make use of this representation. See e.g. [11], Section 2.1 for a complete discussion of this point.

The following objects will be needed in the sequel.

- A (Young) tableau $t$ of shape $(n-m, m)$ is a pair $t=\left(i_{(n-m)} ; j_{(m)}\right)$ of ordered vectors of the type $i_{(n-m)}=\left(i_{1}, \ldots, i_{n-m}\right), j_{(m)}=\left(j_{n-m+1}, \ldots, j_{n}\right)$ such that $\left\{i_{1}, \ldots, i_{n-m}, j_{n-m+1}, \ldots, j_{n}\right\}=[n]$, that is, the union of the entries of $i_{(n-m)}$ and $j_{(m)}$ coincides with the first $n$ integers (with no repetitions).

- The set of the columns of the tableau $t=\left(i_{(n-m)} ; j_{(m)}\right)$, noted $\left\{C_{1}, \ldots, C_{n-m}\right\}$, is the collection of (i) the ordered pairs

$$
C_{1}=\left(i_{1}, j_{n-m+1}\right), \ldots, C_{m}=\left(i_{m}, j_{n}\right)
$$

(that is, the pairs composed of the first $m$ entries of $i_{(n-m)}$ and the entries of $j_{(m)}$ ), and (ii) the remaining singletons of $i_{(n-m)}$, that is,

$$
C_{m+1}=i_{m+1}, \ldots, C_{n-m}=i_{n-m} .
$$

- For $l=1, \ldots, n$, we write $V^{(n-l, l)}$ to indicate the class of the $\left(\begin{array}{l}n \\ l\end{array}\right)$ subsets of $[n]$ of size equal to $l$. This slightly unusual notation has been chosen in order to stress the connection between the set $V^{(n-l, l)}$ and 
the $\mathfrak{S}_{n}$-modules $M^{(n-l, l)}(l \leq m)$ to be defined below. The elements of $V^{(n-l, l)}$ are denoted by $\mathbf{a}_{(l)}$, $\mathbf{b}_{(l)}, \mathbf{i}_{(l)}, \mathbf{j}_{(l)}, \ldots$, and so on.

- A tabloid of shape $(n-m, m)$ is a two-block partition of the set $[n]$, of the type

$$
\gamma=\left\{\mathbf{a}_{(n-m)} ; \mathbf{b}_{(m)}\right\}=\left\{\left\{a_{1}, \ldots, a_{n-m}\right\} ;\left\{b_{n-m+1}, \ldots, b_{n}\right\}\right\} .
$$

Of course, a tabloid $\gamma$ of shape $(n-m, m)$ as in (2.3) is completely determined by the specification of set $\mathbf{b}_{(m)}=\left\{b_{n-m+1}, \ldots, b_{n}\right\} \in V^{(n-m, m)}$; to emphasize this dependence, we shall sometimes write $\gamma=\gamma\left(\mathbf{b}_{(m)}\right)$. Note that the mapping $\mathbf{b}_{(m)} \mapsto \gamma\left(\mathbf{b}_{(m)}\right)$ is a bijection between $V^{(n-m, m)}$ and the class of all tabloids of shape $(n-m, m)$.

- Given a tableau $t=\left(i_{(n-m)} ; j_{(m)}\right)$ of shape $(n-m, m)$, we write $\{t\}=\left\{\mathbf{i}_{(n-m)} ; \mathbf{j}_{(m)}\right\}$ (observe the boldface!) to indicate the tabloid defined by $\mathbf{i}_{(n-m)}=\left\{i_{1}, \ldots, i_{n-m}\right\}$ and $\mathbf{j}_{(m)}=\left\{j_{n-m+1}, \ldots, j_{n}\right\}$. In other words, $\{t\}$ is obtained as the two-block partition composed of the collection of the entries of $i_{(n-m)}$ and the collection of the entries of $j_{(m)}$. With the notation introduced at the previous point, one has that $\{t\}=\gamma\left(\mathbf{j}_{(m)}\right)$.

Example 2.2. Let $n=5$ and $m=2$. Then, a tableau of shape $(3,2)$ is $t=\left(i_{(3)} ; j_{(2)}\right)$, where $i_{(3)}=(2,1,3)$ and $j_{(2)}=(5,4)$. The columns of $t$ are $C_{1}=(2,5), C_{2}=(1,4)$ and $C_{3}=3$. The associated tabloid is $\{t\}=\left\{\mathbf{i}_{(3)} ; \mathbf{j}_{(2)}\right\}$, where $\mathbf{i}_{(3)}=\{1,2,3\} \in V^{(2,3)}$ and $\mathbf{j}_{(2)}=\{4,5\} \in V^{(3,2)}$.

\subsection{Actions of $\mathfrak{S}_{n}$}

Fix as before $n \geq 2$ and $1 \leq m \leq n / 2$.

$\underline{\text { Actions on tableaux }}$. For every $x \in \mathfrak{S}_{n}$ and every tableaux $t=\left(i_{(n-m)} ; j_{(m)}\right)$, the action of $x$ on $t$ is defined as follows:

$$
x t=\left(x i_{(n-m)} ; x j_{(m)}\right),
$$

where $x i_{(n-m)}=\left(x\left(i_{1}\right), \ldots, x\left(i_{n-m}\right)\right)$ and $x j_{(m)}=\left(x\left(j_{n-m+1}\right), \ldots, x\left(j_{n}\right)\right)$.

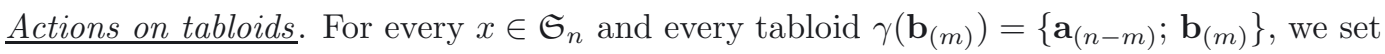

$$
\begin{aligned}
x \gamma\left(\mathbf{b}_{(m)}\right) & =x\left\{\left\{a_{1}, \ldots, a_{n-m}\right\} ;\left\{b_{n-m+1}, \ldots, b_{n}\right\}\right\} \\
& =\left\{\left\{x\left(a_{1}\right), \ldots, x\left(a_{n-m}\right)\right\} ;\left\{x\left(b_{n-m+1}\right), \ldots, x\left(b_{n}\right)\right\}\right\} .
\end{aligned}
$$

In particular, for every tableau $t$, one has $x\{t\}=\{x t\}$.

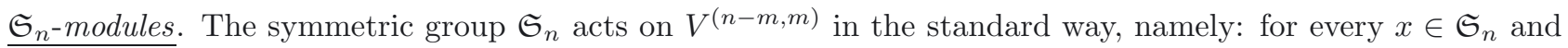
$\overline{\text { for every } \mathbf{j}_{(m)}}=\left\{j_{1}, \ldots, j_{m}\right\} \in V^{(n-m, m)}$,

$$
x \mathbf{j}_{(m)}=\left\{x\left(j_{1}\right), \ldots, x\left(j_{m}\right)\right\} .
$$

Remark 2.3. By combining the above introduced notational conventions, one sees that, for every $x \in \mathfrak{S}_{n}$ and for every $\mathbf{j}_{(m)}=V^{(n-m, m)}$,

$$
x \gamma\left(\mathbf{j}_{(m)}\right)=\gamma\left(x \mathbf{j}_{(m)}\right),
$$

that is, $x$ transforms the tabloid generated by $\mathbf{j}_{(m)}$ into the tabloid generated by $x \mathbf{j}_{(m)}$. Also, if $t=\left(i_{(n-m)} ; j_{(m)}\right)$, then, for every $x \in \mathfrak{S}_{n}$,

$$
x\{t\}=\{x t\}=x \gamma\left(\mathbf{j}_{(m)}\right)=\gamma\left(x \mathbf{j}_{(m)}\right) .
$$


The complex vector space of all complex-valued functions on $V^{(n-m, m)}$ is written $L\left(V^{(n-m, m)}\right)$. Plainly, the space $L\left(V^{(n-m, m)}\right)$ has dimension $\left(\begin{array}{l}n \\ m\end{array}\right)$, and a basis of $L\left(V^{(n-m, m)}\right)$ is given by the collection $\left\{\mathbf{1}_{\mathbf{j}_{(m)}}: \mathbf{j}_{(m)} \in\right.$ $\left.V^{(n-m, m)}\right\}$, where $\mathbf{1}_{\mathbf{j}_{(m)}}\left(\mathbf{k}_{(m)}\right)=1$ if $\mathbf{k}_{(m)}=\mathbf{j}_{(m)}$ and $\mathbf{1}_{\mathbf{j}_{(m)}}\left(\mathbf{k}_{(m)}\right)=0$ otherwise. The group $\mathfrak{S}_{n}$ acts on $L\left(V^{(n-m, m)}\right)$ as follows: for $x \in \mathfrak{S}_{n}, \mathbf{k}_{(m)} \in V^{(n-m, m)}$ and $f \in L\left(V^{(n-m, m)}\right)$,

$$
\begin{aligned}
x f\left(\mathbf{k}_{(m)}\right) & =f\left(x^{-1} \mathbf{k}_{(m)}\right), \text { so that, in particular, } \\
x \mathbf{1}_{\mathbf{j}_{(m)}} & =\mathbf{1}_{x \mathbf{j}_{(m)}}, \quad \mathbf{j}_{(m)} \in V^{(n-m, m)} .
\end{aligned}
$$

When endowed with the action $(2.7)$, the set $L\left(V^{(n-m, m)}\right)$ carries a representation of $\mathfrak{S}_{n}$. In this case, we say that $L\left(V^{(n-m, m)}\right)$ is the permutation module associated with $(n-m, m)$, and we use the customary notation $L\left(V^{(n-m, m)}\right)=M^{(n-m, m)}($ see $[11]$, Sect. 2.1).

Remark 2.4. Our definition of the permutation modules $M^{(n-m, m)}$ slightly differs from the one given e.g. in [11] Definition 2.1.5. Indeed, we define $M^{(n-m, m)}$ as the vector space spanned by all indicators of the type $\mathbf{1}_{\mathbf{j}_{(m)}}, \mathbf{j}_{(m)} \in V^{(n-m, m)}$, endowed with the action (2.7), whereas in the above quoted reference $M^{(n-m, m)}$ is the space of all formal linear combinations of tabloids of shape $(n-m, m)$, endowed with the canonical extension of the action (2.5). The two definitions are equivalent, in the sense that they give rise to two isomorphic $\mathfrak{S}_{n^{-}}$ modules. We will see that the definition of $M^{(n-m, m)}$ chosen in this paper allows a more transparent connection with the theory of $U$-statistics based on random permutations.

\subsection{A decomposition of $M^{(n-m, m)}$}

We recall that the dual of $\mathfrak{S}_{n}$ coincides with the set $\left\{\left[S^{\lambda}\right]: \lambda \vdash n\right\}$, where $\left[S^{\lambda}\right]$ is the equivalence class of all irreducible representations of $\mathfrak{S}_{n}$ that are equivalent to a Specht module of index $\lambda$ (see again [11], Sect. 2.1). For every $\lambda \vdash n$, we will denote by $\chi^{\lambda}$ the character associated with the class $\left[S^{\lambda}\right]$, whereas $D_{\lambda}$ is the associate dimension. Observe that $\chi^{\lambda} \in \mathbb{Z}$ for every $\lambda$ (see e.g. [13], Sect. 13.1), and $D_{\lambda}$ equals the number of standard tableaux (that is, tableaux with increasing rows and columns) of shape $\lambda$. In particular $D_{(n-1,1)}=n-1$ (see [11], Sect. 2.5).

The next result ensures that the module $M^{(n-m, m)}$ is reducible. This fact is well-known (see e.g. [8], example 14.4 (p. 52) or [5] (pp. 134-139)), and a proof is added here for the sake of completeness.

Proposition 2.5. There exists a unique decomposition of $M^{(n-m, m)}$ of the type

$$
M^{(n-m, m)}=K_{0}^{(n-m, m)} \oplus K_{1}^{(n-m, m)} \oplus \cdots \oplus K_{m}^{(n-m, m)} .
$$

Where the vector spaces (endowed with the action of $\mathfrak{S}_{n}$ described in (2.7)) $K_{l}^{(n-m, m)}$ are such that $K_{0}^{(n-m, m)} \in$ $\left[S^{(n)}\right]$, and $K_{l}^{(n-m, m)} \in\left[S^{(n-l, l)}\right], l=1, \ldots, m$.

Proof. It is sufficient to prove that

$$
M^{(n-m, m)} \cong S^{(n)} \oplus \bigoplus_{l=1}^{m} S^{(n-l, l)},
$$

where " $\cong "$ indicates equivalence between representations of $\mathfrak{S}_{n}$. According Young's rule (see e.g. [11], Thm. 2.11.2), we know that

$$
M^{(n-m, m)} \cong S^{(n)} \oplus \bigoplus_{l=1}^{m} K_{n, l, m} S^{(n-l, l)},
$$

where the integers $K_{n, l, m}$ (known as Kostka numbers) count the number of generalized semistandard tableaux of shape $(n-l, l)$ and type $(n-m, m)$. This is equivalent to saying $K_{n, l, m}$ counts the ways of arranging $n-m$ 
copies of 1 and $m$ copies of 2 in a Ferrer diagram of shape $(n-l, l)$, in such a way that the rows of the diagram are weakly increasing and the columns are strictly increasing. Since there is just one way of doing this, one infers that $K_{n, l, m}=1$, and the proof is concluded.

Remark 2.6. (i) (Definition of two-block Specht modules.) For the sake of completeness, we recall here the definition of the modules $S^{(n)}$ and $S^{(n-m, m)}, 1 \leq m \leq n / 2$. First of all, one has that $S^{(n)}=\mathbb{C}$, and therefore $\left[S^{(n)}\right]$ is the class of representations of $\mathfrak{S}_{n}$ that are equivalent to the trivial representation. Now fix $1 \leq m \leq n / 2$. For every tableau $t=\left(i_{(n-m)} ; j_{(m)}\right)$, define the columns $C_{1}, \ldots, C_{n-m}$ according to (2.1) and (2.2). Then, (a) for every $l=1, \ldots, m$, write $\kappa_{C_{l}}$ for the formal operator

$$
\kappa_{C_{l}}=\mathrm{Id} .-\left(i_{l} \rightarrow j_{l}\right)
$$

where $\left(i_{l} \rightarrow j_{l}\right)$ indicates the element of $\mathfrak{S}_{n}$ given by the transposition sending $i_{l}$ to $j_{l}$, and (b) define the composed operator $\kappa_{t}=\kappa_{C_{1}} \kappa_{C_{2}} \ldots \kappa_{C_{m}}$. Then, the Specht module of shape $(n-m, m)$ is the $\mathfrak{S}_{n}$-invariant subspace of $M^{(n-m, m)}$ spanned by the elements of the type

$$
\kappa_{t} \mathbf{1}_{\mathbf{j}_{(m)}}, \quad \text { where } t=\left(i_{(n-m)} ; j_{(m)}\right) \text { is a tableau; }
$$

note that, in the formula $(2.9), t$ and $\mathbf{j}_{(m)}$ are related by the fact that $t=\left(i_{(n-m)} ; j_{(m)}\right)$, and $\{t\}=\left\{\mathbf{i}_{(n-m)} ; \mathbf{j}_{(m)}\right\}$. (ii) Consider for instance the case $n=6$ and $m=2$, and select the tableau $t=\{(1,2,3,4) ;(5,6)\}$. One has that $\mathbf{j}_{(2)}=\{5,6\}$,

$$
\kappa_{t}=(\mathrm{Id} .-(1 \rightarrow 5))(\mathrm{Id} .-(2 \rightarrow 6)),
$$

and one deduces that an element of $S^{(4,2)}$ is given by

$$
\kappa_{t} \mathbf{1}_{\mathbf{j}_{(2)}}=\mathbf{1}_{\{5,6\}}-\mathbf{1}_{\{1,6\}}-\mathbf{1}_{\{5,2\}}+\mathbf{1}_{\{1,2\}}
$$

(iii) By recurrence, one deduces from Proposition 2.5 that the dimension of $K_{l}^{(n-m, m)}$, and therefore of $S^{(n-l, l)}$, is $D_{(n-l, l)}=\left(\begin{array}{c}n \\ l\end{array}\right)-\left(\begin{array}{c}n \\ l-1\end{array}\right), l \leq n / 2$.

(iv) From the previous discussion, we infer that $K_{0}^{(n-m, m)}=S^{(n)}=\mathbb{C}$.

\section{UNIFORM RANDOM PERMUTATIONS}

Fix $n \geq 2$. We consider a uniform random permutation $X$ of $[n]$. This means that $X=X(\omega)$ is a random element with values in $\mathfrak{S}_{n}$, defined on some finite probability space $(\Omega, \mathcal{F}, \mathbf{P})$ and such that, $\forall x \in \mathfrak{S}_{n}$, $\mathbf{P}(X=x)=(n !)^{-1}$. For $1 \leq m \leq n / 2$ as before, we will write $X_{(m)}(\omega)=(X(1), \ldots, X(m))(\omega)$, and also, for every $y \in \mathfrak{S}_{n},(X y)_{(m)}=\{X y(1), \ldots, X y(m)\}$. Observe that $X y$ indicates the product of the deterministic permutation $y$ with the random permutation $X$. It is clear that $X_{(m)}$ is an exchangeable vector, having the law of the first $m$ extractions without replacement from the set $[n]$ (see e.g. Aldous [1] for any unexplained notion about exchangeability). A random variable $T$ is called a (complex-valued) symmetric statistic of $X_{(m)}$ if $T$ has the form

$$
T=f(\{X(1), \ldots, X(m)\}), \quad \text { for some } f \in L\left(V^{(n-m, m)}\right)
$$

In other words, a symmetric statistic is a random variable deterministically depending on the realization of $X_{(m)}$ as a non-ordered set. Note that, by a slight abuse of notation, in what follows we will write $f(\{X(1), \ldots$, $X(m)\})=f\left(X_{(m)}\right)$ (other analogous conventions will be tacitly adopted). 
We also write $L_{s}^{2}\left(X_{(m)}\right)$ to indicate the Hilbert space of symmetric statistics of $X_{(m)}$, endowed with the inner product

$$
\begin{aligned}
\left\langle f_{1}\left(X_{(m)}\right), f_{2}\left(X_{(m)}\right)\right\rangle_{\mathbf{P}} & =\mathbf{E}\left[f_{1}\left(X_{(m)}\right) \overline{f_{2}\left(X_{(m)}\right)}\right] \\
& =\frac{1}{n !} \sum_{x \in \mathfrak{S}_{n}} f_{1}(x\{1, \ldots, m\}) \overline{f_{2}(x\{1, \ldots, m\})} \\
& =\left(\begin{array}{c}
n \\
m
\end{array}\right)^{-1} \sum_{\mathbf{k}_{(m)} \in V^{(n-m, m)}} f_{1}\left(\mathbf{k}_{(m)}\right) \overline{f_{2}\left(\mathbf{k}_{(m)}\right)} .
\end{aligned}
$$

Since the sum in (3.2) runs over the whole set $\mathfrak{S}_{n}$, it is clear that $\langle\cdot, \cdot\rangle_{\mathbf{P}}$ induces a $\mathfrak{S}_{n}$-invariant inner product on $M^{(n-m, m)}$ given by

$$
\left\langle f_{1}, f_{2}\right\rangle_{(n-m, m)}=\left\langle f_{1}\left(X_{(m)}\right), f_{2}\left(X_{(m)}\right)\right\rangle_{\mathbf{P}}, \quad f_{1}, f_{2} \in M^{(n-m, m)} ;
$$

in particular, the $\mathfrak{S}_{n}$-invariance of $\langle\cdot, \cdot\rangle_{(n-m, m)}$ yields that the spaces $K_{i}^{(n-m, m)}$ and $K_{j}^{(n-m, m)}$ are orthogonal with respect to $\langle\cdot, \cdot\rangle_{(n-m, m)}$ for every $0 \leq i \neq j \leq m$.

With every $f \in M^{(n-m, m)}$, we associate the $\mathfrak{S}_{n}$-indexed stochastic process

$$
Z_{f}(x, \omega)=Z_{f}(x):=f\left(x X_{(m)}\right), \quad x \in \mathfrak{S}_{n},
$$

and, for every $\lambda \vdash n$, we define

$$
\begin{aligned}
& Z_{f}^{\lambda}(x, \omega)=Z_{f}^{\lambda}(x):=\frac{D_{\lambda}}{n !} \sum_{g \in \mathfrak{S}_{n}} \chi^{\lambda}(g) f\left(\left(g^{-1} x\right) X_{(m)}\right) \\
& f^{\lambda}\left(\mathbf{l}_{(m)}\right)=\frac{D_{\lambda}}{n !} \sum_{x \in \mathfrak{S}_{n}} \chi^{\lambda}(x) f\left(x^{-1} \mathbf{l}_{(m)}\right), \quad \mathbf{l}_{(m)} \in V^{(n-m, m)},
\end{aligned}
$$

so that $f^{\lambda}\left(X_{(m)}\right)=Z_{f}^{\lambda}(e)$, where $e$ is the identity element in $\mathfrak{S}_{n}$.

The following facts will be used in the subsequent analysis. The proofs are standard and omitted - see e.g. the results from [10] and [13] evoked below for further details.

(a) Since (2.8) holds, $f^{\lambda}=0$ for every $f \in M^{(n-m, m)}$ if and only if $\lambda$ is different from $(n-l, l), l=0, \ldots, m$ (see e.g. [13], Thm. 8, Sect. 2.6) and moreover: $f^{(n)} \in K_{0}^{(n-m, m)}$ and, for every $l=1, \ldots, m, f^{(n-l, l)} \in$ $K_{l}^{(n-m, m)}$ (as defined in (2.8)).

(b) Thanks to exchangeability, for every $f \in M^{(n-m, m)}$ the class

$$
\left\{Z_{f}, Z_{f}^{(n-l, l)}: l=0, \ldots, m\right\},
$$

has a $\mathfrak{S}_{n}$-invariant law, with respect to the canonical action of $\mathfrak{S}_{n}$ on itself $\left(i . e ., x \cdot y=x y, x, y \in \mathfrak{S}_{n}\right)$.

(c) Due to the orthogonality of isotypical spaces (see e.g. (see [6], Thm. 4.4.5, and also [10], Thm. 4-3), for every $x, y \in \mathfrak{S}_{n}, f, h \in M^{(n-m, m)}$ and $0 \leq i \neq j \leq m$,

$$
\begin{aligned}
& \mathbf{E}\left[Z_{f}^{(n-i, i)}(x) \overline{Z_{h}^{(n-j, j)}(y)}\right]=\mathbf{E}\left[f^{(n-i, i)}\left(x X_{(m)}\right) \overline{h^{(n-j, j)}\left(y X_{(m)}\right)}\right] \\
& \mathbf{E}\left[f^{(n-i, i)}\left((X x)_{(m)}\right) \overline{h^{(n-j, j)}\left((X y)_{(m)}\right)}\right]=0,
\end{aligned}
$$

where, here and in the sequel (by a slight abuse of notation) we use the convention $(n-0,0)=(n)$. 
(d) Due to [10], Theorem $4-4$ and point (a) above, for every $x \in \mathfrak{S}_{n}$ and every $f \in M^{(n-m, m)}$,

$$
Z_{f}(x)=Z_{f}^{(n)}(x)+\sum_{l=1}^{m} Z_{f}^{(n-l, l)}(x),
$$

where $Z_{f}^{(n)}(x)=\mathbf{E}\left[Z_{f}(x)\right]=\mathbf{E}\left[f\left(X_{(m)}\right)\right]$. In particular,

$$
f\left(X_{(m)}\right)=\mathbf{E}\left[f\left(X_{(m)}\right)\right]+\sum_{l=1}^{m} f^{(n-l, l)}\left(X_{(m)}\right)
$$

and therefore, for every $f, h \in M^{(n-m, m)}$,

$\mathbf{E}\left[f\left(X_{(m)}\right) \overline{h\left(X_{(m)}\right)}\right]=\mathbf{E}\left[f\left(X_{(m)}\right)\right] \overline{\mathbf{E}\left[h\left(X_{(m)}\right)\right]}+\sum_{l=1}^{m} \mathbf{E}\left[f^{(n-l, l)}\left(X_{(m)}\right) \overline{h^{(n-l, l)}\left(X_{(m)}\right)}\right]$

(e) Due to [10], Theorem $5-1$, for every $0 \leq i \neq j \leq m$ and $f, h \in M^{(n-m, m)}$,

$$
\sum_{x \in \mathfrak{S}_{n}} Z_{f}^{(n-i, i)}(x, \omega) \overline{Z_{h}^{(n-j, j)}(x, \omega)}=\sum_{x \in \mathfrak{S}_{n}} f^{(n-i, i)}\left(x X_{(m)}\right) \overline{h^{(n-j, j)}\left(x X_{(m)}\right)}=0 .
$$

\section{HoefFding SPACES}

We now define a class of subspaces of $L_{s}^{2}\left(X_{(m)}\right)$ (the notation is the same as in [7,9]): $S U_{0}=\mathbb{C}$, and, for $l=1, \ldots, m, S U_{l}$ is the vector subspace generated by the functionals of $X_{(m)}$ of the type

$$
T_{\phi}\left(X_{(m)}\right)=\sum_{\left\{k_{1}, \ldots, k_{l}\right\} \in V^{(m-l, l)}} \phi\left(X\left(k_{1}\right), \ldots, X\left(k_{l}\right)\right)
$$

for some $\phi \in L\left(V^{(n-l, l)}\right)$. A random variable such as (4.1) is called a $U$-statistic based on $X_{(m)}$, with a symmetric kernel $\phi$ of order $l$. One has that $S U_{l} \subset S U_{l+1}$ (see e.g. [9]) and $S U_{m}=L_{s}^{2}\left(X_{(m)}\right)$. The collection of the symmetric Hoeffding spaces associated to $X_{(m)}$, noted $\left\{S H_{l}: l=0, \ldots, m\right\}$ is defined as follows: $S H_{0}=S U_{0}$, and

$$
S H_{l}=S U_{l} \cap S U_{l-1}^{\perp},
$$

where the symbol $\perp$ means orthogonality with respect to the inner product $\langle\cdot, \cdot\rangle_{\mathbf{P}}$ defined in (3.1), so that

$$
L_{s}^{2}\left(X_{(m)}\right)=\bigoplus_{l=0}^{m} S H_{l}
$$

where the direct sum $\bigoplus$ is again in the sense of $\langle\cdot, \cdot\rangle_{\mathbf{P}}$.

Following [3], Section 2, we define the real coefficients

$$
\begin{aligned}
d_{l, j} & =\prod_{r=j}^{l-1} \frac{n-r}{n-r-j}, \quad l=2,3, \ldots, m, 1 \leq j \leq l-1, \\
d_{l, l} & =N_{l, l}=1, \quad l=1, \ldots, m, \\
N_{l, j} & =-\sum_{i=j}^{l-1}\left(\begin{array}{c}
l-j \\
i-j
\end{array}\right) d_{l, i} N_{i, j}, \quad l=2,3, \ldots, m, \quad 1 \leq j \leq l-1 .
\end{aligned}
$$

The following result can be proved by using the content of [3], Section 2, or as a special case of [9], Theorem 11. 
Proposition 4.1. Keep the assumptions and notation of this section. Then, for $l=1, \ldots, m$, the following assertions are equivalent:

(i) $f\left(X_{(m)}\right) \in S H_{l}$;

(ii) there exists $\phi \in L\left(V^{(n-l, l)}\right)$ such that

$$
f\left(X_{(m)}\right)=\sum_{\left\{k_{1}, \ldots, k_{l}\right\} \in V^{(m-l, l)}} \phi\left(X\left(k_{1}\right), \ldots, X\left(k_{l}\right)\right),
$$

and

$$
\mathbf{E}[\phi(X(1), \ldots, X(l)) \mid X(1), \ldots, X(l-1)]=0 .
$$

Moreover, for every $h\left(X_{(m)}\right) \in L_{s}^{2}\left(X_{(m)}\right)$, the orthogonal projection of $h\left(X_{(m)}\right)$ on $S H_{l}, l=1, \ldots, m$, is given by

$$
\operatorname{proj}\left(h\left(X_{(m)}\right) \mid S H_{l}\right)=\sum_{\left\{k_{1}, \ldots, k_{l}\right\} \in V^{(m-l, l)}} \phi_{h}^{(l)}\left(X\left(k_{1}\right), \ldots, X\left(k_{l}\right)\right)
$$

where, for every $\left\{j_{1}, \ldots, j_{l}\right\} \in V^{(n-l, l)}$,

$$
\phi_{h}^{(l)}\left(j_{1}, \ldots, j_{l}\right)=d_{m, l} \sum_{a=1}^{l} N_{l, a} \sum_{1 \leq i_{1}<\ldots<i_{a} \leq l} \mathbf{E}\left[h\left(X_{(m)}\right)-\mathbf{E}\left(h\left(X_{(m)}\right)\right) \mid X(1)=j_{i_{1}}, \ldots, X(a)=j_{i_{a}}\right] .
$$

The kernel $\phi$ of the $U$-statistic $f\left(X_{(m)}\right)$ appearing in (4.3) is said to be completely degenerated. Completely degenerated kernels are related to the notion of weak independence in [9], Theorem 6. Note that, in the above quoted references, the content of Proposition 4.1 is proved for real valued symmetric statistics (the extension of such results to complex random variables is immediate: just consider separately the real and the imaginary parts of each statistic). Formula (4.4) completely characterizes the symmetric Hoeffding spaces associated to $X_{(m)}$ : it can be obtained by recursively applying an appropriate version of the Möbius inversion formula (see e.g. [11], exercise 18, Sect. 5.6), on the lattice of the subsets of $[n]$ (see also [9], Thm. 11, for a generalization of (4.4) to the case of Generalized Urn Sequences). In the next section we state and prove the main result of this note, that is, that the spaces $S H_{l}, l=1, \ldots, m$, admit a further algebraic characterization in terms of Specht modules.

\section{Hoeffding SPACES AND TWO-Blocks Specht MOdules}

\subsection{Main results and some consequences}

The main achievement of this note is the following statement, which is a more precise reformulation of Theorem 1.1, as stated in the Introduction. The proof is deferred to Section 5.2.

Theorem 5.1. Under the above notation and assumptions, for every $f\left(X_{(m)}\right) \in L_{s}^{2}\left(X_{(m)}\right)$ and every $l=$ $0,1, \ldots, m$, the following assertions are equivalent:

(1) $f\left(X_{(m)}\right) \in S H_{l}$;

(2) $f \in K_{l}^{(n-m, m)}$, where the $\mathfrak{S}_{n}$-module $K_{l}^{(n-m, m)}$ is defined through formula (2.8) (in particular, $K_{l}^{(n-m, m)}$ $\left.\in\left[S^{(n-l, l)}\right]\right)$.

We now list some consequences of Theorem 5.1. They can be obtained by properly combining Proposition 4.1 with the five facts (a)-(e), as listed at the end of Section 3. 
Corollary 5.2. Under the above notation and assumptions,

(1) for every $l=1, \ldots, m, f \in M^{(n-m, m)}$ and $\mathbf{i}_{(m)}=\left\{i_{1}, \ldots, i_{m}\right\} \in V^{(n-m, m)}$,

$$
\begin{aligned}
f^{(n-l, l)}\left(\mathbf{i}_{(m)}\right)= & \frac{D_{(n-l, l)}}{n !} \sum_{x \in \mathfrak{S}_{n}} \chi^{(n-l, l)}(x) f\left(x^{-1} \mathbf{i}_{(m)}\right) \\
= & \sum_{\left\{i_{1}, \ldots, i_{l}\right\} \subseteq \mathbf{i}_{(m)}} d_{m, l} \sum_{a=1}^{l} N_{l, a} \\
& \times \sum_{1 \leq s_{1}<\ldots<s_{a} \leq l} \mathbf{E}\left[f\left(X_{(m)}\right)-\mathbf{E}\left(f\left(X_{(m)}\right)\right) \mid X(1)=i_{s_{1}}, \ldots, X(a)=i_{s_{a}}\right],
\end{aligned}
$$

where $D_{(n-l, l)}=\left(\begin{array}{c}n \\ l\end{array}\right)-\left(\begin{array}{c}n \\ l-1\end{array}\right)$.

(2) for every $l=1, \ldots, m$, every symmetric $U$-statistic, based on $X_{(m)}$ and with a completely degenerated kernel of order $l$, has the form (5.1) for some $f \in M^{(n-m, m)}$. It follows that $S H_{l}$ is an irreducible $\mathfrak{S}_{n}$-module, carrying a representation in $\left[S^{(n-l, l)}\right]$.

For instance, by using [11], exercise 5.d (p. 87), we deduce from (5.1) that for every $\mathbf{i}_{(m)}=\left\{i_{1}, \ldots, i_{m}\right\} \in$ $V^{(n-m, m)}$ and $f \in M^{(n-m, m)}$,

$$
\begin{aligned}
& \frac{n-1}{n !} \sum_{x \in \mathfrak{S}_{n}}\{(\text { number of fixed points of } x)-1\} \times f\left(x \mathbf{i}_{(m)}\right) \\
= & \prod_{r=1}^{m-1} \frac{n-r}{n-r-1} \sum_{s=1}^{m} \mathbf{E}\left[f\left(X_{(m)}\right)-\mathbf{E}\left(f\left(X_{(m)}\right)\right) \mid X(1)=i_{s}\right] .
\end{aligned}
$$

The next result gives an algebraic explanation of a property of degenerated $U$-statistics, already pointed out - in the more general framework of Generalized Urn Sequences - in [9] (Cor. 9). Basically, it states that the orthogonality, between two completely degenerated $U$-statistics of different orders, is preserved after shifting one of the two arguments. It can be useful when determining the covariance between two $U$-statistics based on two urn sequences of different lengths.

Corollary 5.3. Let $f, h \in M^{(n-m, m)}$ be such that $f\left(X_{(m)}\right) \in S H_{j}$ and $h\left(X_{(m)}\right) \in S H_{l}$ for some $1 \leq j \neq$ $l \leq m$. Consider moreover an element $\mathbf{k}_{(m)}=\left\{k_{1}, \ldots, k_{m}\right\} \in V^{(n-m, m)}$ such that, for some $r=0, \ldots, m$, Card $\left(\mathbf{k}_{(m)} \cap\{1, \ldots, m\}\right)=r$, and note $X_{(m)}^{\prime}=\left(X\left(k_{1}\right), \ldots, X\left(k_{m}\right)\right)$. Then,

$$
\mathbf{E}\left(f\left(X_{(m)}\right) \overline{h\left(X_{(m)}^{\prime}\right)}\right)=0 .
$$

Proof. Due to the exchangeability of the vector $(X(1), \ldots, X(n))$, we can assume, without loss of generality, that

$$
\mathbf{k}_{(m)}=\{1, \ldots, r, m+1, \ldots, 2 m-r\} .
$$

Now introduce the permutation (written as a product of translations)

$$
y=(r+1 \rightarrow m+1)(r+2 \rightarrow m+2) \cdots(m \rightarrow 2 m-r)
$$

and note that

$$
\mathbf{E}\left(f\left(X_{(m)}\right) \overline{h\left(X_{(m)}^{\prime}\right)}\right)=\mathbf{E}\left(f\left(X_{(m)}\right) \overline{h\left((X y)_{(m)}\right)}\right),
$$

so that the conclusion derives immediately from formula (3.6), by setting $x=e$ and $y$ as in (5.3). 


\subsection{Remaining proofs}

The key of the proof of Theorem 5.1 is nested in the following lemma.

Lemma 5.4. Let the previous notation prevail. Then,

(1) for each $l=1, \ldots, m$, a basis of $S U_{l}$ is given by the set of random variables

$$
\left\{\eta_{\mathbf{i}_{(l)}}\left(X_{(m)}\right): \mathbf{i}_{(l)} \in V^{(n-l, l)}\right\},
$$

where, for each $\mathbf{k}_{(m)} \in V^{(n-m, m)}$,

$$
\eta_{\mathbf{i}_{(l)}}\left(\mathbf{k}_{(m)}\right)= \begin{cases}1 & \text { if } \mathbf{i}_{(l)} \subseteq \mathbf{k}_{(m)} \\ 0 & \text { otherwise }\end{cases}
$$

(2) for each $l=1, \ldots, m$, the restriction of the action (2.7) of $\mathfrak{S}_{n}$ to the vector subspace of $M^{(n-m, m)}$ generated by the set $\left\{\eta_{\mathbf{i}_{(l)}}: \mathbf{i}_{(l)} \in V^{(n-l, l)}\right\}$, defined in (5.4), is equivalent to the action carried by the $\mathfrak{S}_{n}$-module $M^{(n-l, l)}$.

Proof. Fix $l=1, \ldots, m$, and observe that, for every $\mathbf{i}_{(l)} \in V^{(n-l, l)}$,

$$
\eta_{\mathbf{i}_{(l)}}\left(X_{(m)}\right)=\sum_{\left\{k_{1}, \ldots, k_{l}\right\} \in V^{(m-l, l)}} \mathbf{1}_{\mathbf{i}_{(l)}}\left(\left\{X\left(k_{1}\right), \ldots, X\left(k_{l}\right)\right\}\right),
$$

so that the first part of the statement follows from the definition of $S U_{l}$, and the fact that every $\phi \in V^{(m-l, l)}$ is a linear combination of functions of the type $\mathbf{1}_{\mathbf{i}_{(l)}}(\cdot)$. To prove the second part, first recall that a basis of the $\mathfrak{S}_{n}$-module $M^{(n-l, l)}$ is given by the set $\left\{\mathbf{1}_{\mathbf{i}_{(l)}}(\cdot): \mathbf{i}_{(l)} \in V^{(n-l, l)}\right\}$, and that the action of $\mathfrak{S}_{n}$ on $M^{(n-l, l)}$ is completely described by the action

$$
x \mathbf{1}_{\mathbf{i}_{(l)}}=\mathbf{1}_{x \mathbf{i}_{(l)}} .
$$

We can therefore construct a $\mathfrak{S}_{n}$-isomorphism between $\left\{\eta_{\mathbf{i}_{(l)}}: \mathbf{i}_{(l)} \in V^{(n-l, l)}\right\}$ and $M^{(n-l, l)}$ by linearly extending the mapping

$$
\tau\left(\eta_{\mathbf{i}_{(l)}}\right)=\mathbf{1}_{\mathbf{i}_{(l)}}, \quad \mathbf{i}_{(l)} \in V^{(n-l, l)},
$$

and by observing that, for every $\mathbf{k}_{(m)} \in V^{(n-m, m)}, \mathbf{i}_{(l)} \in V^{(n-l, l)}$ and $x \in \mathfrak{S}_{n}$,

$$
x \eta_{\mathbf{i}_{(l)}}\left(\mathbf{k}_{(m)}\right)=\eta_{\mathbf{i}_{(l)}}\left(x^{-1} \mathbf{k}_{(m)}\right)=\eta_{x \mathbf{i}_{(l)}}\left(\mathbf{k}_{(m)}\right) .
$$

This concludes the proof.

End of the proof of Theorem 5.1. Since $S U_{0}=S H_{0}=K_{0}^{(n-m, m)}=\mathbb{C}$, the relation between representations

$$
M^{(n-l, l)} \cong S^{(n)} \oplus S^{(n-1,1)} \oplus \cdots \oplus S^{(n-l, l)}, \quad \forall l=1, \ldots, m,
$$

along with Lemma 5.4, implies that the restriction of the action (2.7) of $\mathfrak{S}_{n}$ to those $f \in L\left(V^{(n-m, m)}\right)$ such that $f\left(X_{(m)}\right) \in S H_{l}$ is an element of $\left[S^{(n-l, l)}\right]$. This yields that each one of the $m+1$ summands in the decomposition

$$
M^{(n-m, m)}=\mathbb{C} \oplus \bigoplus_{l=1}^{m}\left\{f: f\left(X_{(m)}\right) \in S H_{l}\right\}
$$

is an irreducible $\mathfrak{S}_{n}$-submodule of $M^{(n-m, m)}$. Since the decomposition $(2.8)$ of $M^{(n-m, m)}$ is unique, this gives

$$
\left\{f: f\left(X_{(m)}\right) \in S H_{l}\right\}=K_{l}^{(n-m, m)},
$$

as required. 
Acknowledgements. We are grateful to O. El-Dakkak for useful comments.

\section{REFERENCES}

[1] D.J. Aldous, Exchangeability and related topics. École d'été de Probabilités de Saint-Flour XIII. LNM 1117, Springer, New York (1983).

[2] M. Bloznelis, Orthogonal decomposition of symmetric functions defined on random permutations. Combin. Probab. Comput. 14 (2005) 249-268.

[3] M. Bloznelis and F. Götze, Orthogonal decomposition of finite population statistics and its applications to distributional asymptotics. Ann. Stat. 29 (2001) 353-365.

[4] M. Bloznelis and F. Götze, An Edgeworth expansion for finite population statistics. Ann. Probab. 30 (2002) $1238-1265$.

[5] P. Diaconis, Group Representations in Probability and Statistics. IMS Lecture Notes - Monograph Series 11, Hayward, California (1988).

[6] J.J. Duistermaat and J.A.C. Kolk, Lie groups. Springer-Verlag, Berlin-Heidelberg-New York (1997).

[7] O. El-Dakkak and G. Peccati, Hoeffding decompositions and urn sequences. Ann. Probab. 36 (2008) 2280-2310.

[8] G.D. James, The representation theory of the symmetric groups. Lecture Notes in Math. 682, Springer-Verlag, BerlinHeidelberg-New York (1978).

[9] G. Peccati, Hoeffding-ANOVA decompositions for symmetric statistics of exchangeable observations. Ann. Probab. 32 (2004) $1796-1829$.

[10] G. Peccati and J.-R. Pycke, Decompositions of stochastic processes based on irreducible group representations. Theory Probab. Appl. 54 (2010) 217-245.

[11] B.E. Sagan, The Symmetric Group. Representations, Combinatorial Algorithms and Symmetric Functions, 2nd edition. Springer, New York (2001).

[12] R.J. Serfling, Approximation Theorems of Mathematical Statistics. Wiley, New York (1980).

[13] J.-P. Serre, Linear representations of finite groups, Graduate Texts Math. 42, Springer, New York (1977).

[14] L. Zhao and X. Chen, Normal approximation for finite-population U-statistics. Acta Math. Appl. Sinica 6 (1990) $263-272$. 